\title{
Sparse Fault-Tolerant Spanners for Doubling Metrics with Bounded Hop-Diameter or Degree
}

\author{
T-H. Hubert Chan, Mingfei Li, and Li Ning \\ The University of Hong Kong
}

\begin{abstract}
We study fault-tolerant spanners in doubling metrics. A subgraph $H$ for a metric space $X$ is called a $k$-vertex-fault-tolerant $t$-spanner $((k, t)$-VFTS or simply $k$-VFTS), if for any subset $S \subseteq X$ with $|S| \leq k$, it holds that $d_{H \backslash S}(x, y) \leq t \cdot d(x, y)$, for any pair of $x, y \in X \backslash S$.

For any doubling metric, we give a basic construction of $k$-VFTS with stretch arbitrarily close to 1 that has optimal $O(k n)$ edges. In addition, we also consider bounded hop-diameter, which is studied in the context of fault-tolerance for the first time even for Euclidean spanners. We provide a construction of $k$-VFTS with bounded hop-diameter: for $m \geq 2 n$, we can reduce the hop-diameter of the above $k$-VFTS to $O(\alpha(m, n))$ by adding $O(\mathrm{~km})$ edges, where $\alpha$ is a functional inverse of the Ackermann's function.

Finally, we construct a fault-tolerant single-sink spanner with bounded maximum degree, and use it to reduce the maximum degree of our basic $k$-VFTS. As a result, we get a $k$-VFTS with $O\left(k^{2} n\right)$ edges and maximum degree $O\left(k^{2}\right)$.
\end{abstract}

\section{Introduction}

A metric space $(X, d)$ can be represented by a complete graph $G=(X, E)$, where the edge weight $w(e)$ on an edge $e=\{x, y\}$ is $d(x, y)$. A $t$-spanner of $X$, is a weighted subgraph $H=\left(X, E^{\prime}\right)$ of $G$ that preserves all pairwise distance within a factor of $t$, i.e., $d_{H}(x, y) \leq t \cdot d(x, y)$ for all $x, y \in X$. Here, $d_{H}(x, y)$ denotes the shortest-path distance between $x$ and $y$ in $H$. The factor $t$ is called the stretch of $H$. A path between $x$ and $y$ in $H$ with length at most $t \cdot d(x, y)$ is called a t-spanner path. Spanners have been studied extensively since the mid-eighties (see $[2,8,1,16,12,3,4,10]$ and the references therein; also refer to [15] for an excellent survey).

Spanners are important structures, as they enable approximation of a metric space in a much more economical form. One natural requirement is that spanners should be sparse, ideally with the number of edges being linear in the number of points in the metric space. In addition, for some applications, it might also be required that a spanner should have small maximum degree, or a small hopdiameter, i.e., every pair of points $x$ and $y$ should be connected by a $t$-spanner path with small number of edges.

In many applications of spanners, we want our spanner to be robust to failures, meaning that even when some of the points in the spanner fail, the remaining part is still a $t$-spanner. Formally, given $1 \leq k \leq n-2$, a spanner $H$ of $X$ is 
called a $k$-vertex-fault-tolerant $t$-spanner $((k, t)$-VFTS or simply $k$-VFTS if the stretch $t$ is clear from context), if for any subset $S \subseteq X$ with $|S| \leq k, H \backslash S$ is a $t$-spanner for $X \backslash S$.

The notion of fault-tolerant spanners was introduced by Levcopoulos et al. [13] in the context of Euclidean spanners (the special case when $X$ is a finite subset of low dimensional Euclidean space and $\left.d(x, y)=\|x-y\|_{2}\right)$. They presented an algorithm that constructs Euclidean $(k, 1+\epsilon)$-VFTS with $O\left(k^{2} n\right)$ edges. This result was later improved by Lukovszki in [14]. They provided two constructions of $(k, 1+\epsilon)$-VFTS: one with optimal $O(k n)$ edges, and the other with $O\left(k^{2} n\right)$ edges and maximum degree $O\left(k^{2}\right)$. There has also been research on the trade-off between maximum degree and weight in fault-tolerant Euclidean spanners [13, 7], and fault-tolerant spanners for general graphs [6,9].

In this paper, we study fault-tolerant spanners for doubling metrics. The doubling dimension of a metric space $(X, d)$, denoted by $\operatorname{dim}(X)$ (or $\operatorname{dim}$ when the context is clear), is the smallest value $\rho$ such that every ball in $X$ can be covered by $2^{\rho}$ balls of half the radius [11]. A metric space is called doubling, if its doubling dimension is bounded by some constant. Doubling dimension is a generalization of Euclidean dimension to arbitrary metric spaces, as the space $\mathbb{R}^{T}$ equipped with $\ell_{p}$-norm has doubling dimension $\Theta(T)$ [11]. Spanners for doubling metrics have been studied in $[12,3,4,10,16]$.

\subsection{Our Results and Techniques}

Basic spanner with small number of edges. Our first result is a construction of $(k, 1+\epsilon)$-VFTS for doubling metrics with $O(k n)$ edges. Note that the size is optimal up to a constant factor [14].

Theorem $1((k, 1+\epsilon)$-VFTS with $O(k n)$ Edges $)$. Let $(X, d)$ be a doubling metric with $n$ points and let $0<\epsilon<\frac{1}{2}$ be a constant. Given $1 \leq k \leq n-2$, there exists $a(k, 1+\epsilon)-V F T S$ of $X$ with $\epsilon^{-O(\operatorname{dim})} \cdot k n$ edges.

Our technique of the basic $k$-VFTS construction is an extension of that in [3]. Specifically, we give a $k$-fault-tolerant version of the hierarchical nets and net-trees used in [3], which guarantee that even under the failure of at most $k$ points, for any functioning point $x$ and level $i$, there exists a net-tree in which there is a path with length at most $O\left(2^{i}\right)$ between $x$ and some level- $i$ net point. We also add cross edges between net points at the same level that are reasonably close. Then, we show that under the failure of at most $k$ points, a $(1+\epsilon)$-spanner path between $x$ and $y$ can be formed by first climbing from $x$ to some net point $x^{\prime}$ at an appropriate level $i$, then going along a cross edge $\left\{x^{\prime}, y^{\prime}\right\}$, and finally going from $y^{\prime}$ down to $y$.

The upper bound on the number of edges in the spanner is established in a way similar to [3] by carefully assigning a direction to each edge, and then showing that the out-degree of each point is bounded by $O(k)$. However, we simplify and improve the analysis because in our case cross edges can be added between arbitrarily close points. 
Spanners with small hop-diameters. We also consider the hop-diameter, which is studied in the context of fault-tolerance for the first time even for Euclidean spanners. The $k$-vertex-fault-tolerant hop-diameter is defined as follows.

Definition 1 ( $k$-Vertex-Fault-Tolerant Hop-Diameter). Let $H$ be $a(k, t)$ VFTS for the metric space $(X, d)$. The $k$-vertex-fault-tolerant hop-diameter (or simply hop-diameter) of $H$ is at most $D$, if for any set of points $S \subseteq X$ with $|S| \leq k$, there exists a $t$-spanner path in $H \backslash S$ with at most $D$ edges (hops) between every pair of $x, y \in X$.

We show that by adding a few extra edges to our basic $k$-VFTS, we can significantly reduce its hop-diameter.

Theorem $2((k, 1+\epsilon)$-VFTS with Small Hop-Diameter). Let $m \geq 2 n$. We can add $O(\mathrm{~km})$ extra edges to the spanner in Theorem 1 to get a $(k, 1+\epsilon)$ VFTS with hop-diameter at most $O(\alpha(m, n))$, where $\alpha$ is the functional inverse of Ackermann's function.

The technique of reducing the hop-diameter is similar to that in [4]. Let $H$ be the spanner in Theorem 1. Recall that when the points in $S$ fail, the $(1+\epsilon)$ spanner path in $H \backslash S$ between any two points of $x$ and $y$ is the concatenation of a path $P_{1}$ in some net-tree $T_{1}$, the cross edge $\left\{x^{\prime}, y^{\prime}\right\}$ and a path $P_{2}$ in some net-tree $T_{2}$. We add edges to net-trees to shortcut the paths $P_{1}$ and $P_{2}$, and hence obtain a spanner with small hop-diameter.

Spanners with bounded degree. We also give a construction of $(k, t)$-VFTS with bounded maximum degree. This is achieved with a sacrifice of increasing the number of edges. The result matches the state-of-the-art result of the boundeddegree Euclidean $(k, 1+\epsilon)$-VFTS in [14].

Theorem $3((k, 1+\epsilon)$-VFTS with Bounded Degree). Let $(X, d)$ be a doubling metric with $n$ points and let $0<\epsilon<1$ be a constant. Given $1 \leq k \leq n-2$, there exists a $(k, 1+\epsilon)$-VFTS with $\epsilon^{-O(\operatorname{dim})} \cdot k^{2} n$ edges and maximum degree $\epsilon^{-O(\operatorname{dim})} \cdot k^{2}$.

In [3], it is shown how to reduce the maximum degree of net-tree based spanners for doubling metrics. This is achieved by replacing some cross edges with inter-level edges. As a result, the end points of a replaced cross edge $\{u, v\}$ are connected by a path $\left\{u, w_{1}, w_{2}, \ldots, w_{i}, v\right\}$, with approximately the same length. However, in the context of fault-tolerance, some of the $w_{j}$ 's might fail, and it is unclear how to make this procedure resilient to failures.

However, we note that the degree-reduction techniques similar to those in $[1$, 14] can be applied. Recall that the edges of the $k$-VFTS in Theorem 1 can be directed such that the out-degree of each point is bounded by $O(k)$. To reduce the in-degrees, for each point $x$, we replace the star consisting of $x$ and edges going into $x$ with a $k$-vertex-fault-tolerant single-sink spanner that approximately preserves the distances to $x$, and has maximum degree $O(k)$.

We show how to construct $k$-vertex-fault-tolerant single-sink spanners with bounded degree for doubling metrics. The construction for those in Euclidean 
space is based on $\Theta$-graphs $[1,14]$. We provide a novel technique called ringpartition, which can be seen as a replacement for the $\Theta$-graph in doubling metrics. Given a specific root point from $X$, the metric space $X$ is partitioned into rings centered at the root with geometrically increasing radii, and each ring is further partitioned into small clusters. From each cluster, we select some portals, which are connected to the root by short paths. In addition, points in each cluster are connected to the portals with short paths as well. As a result, every point's distance to the root is approximately preserved.

\subsection{Preliminaries}

For any positive integer $m$, we denote $[m]:=\{1,2, \ldots, m\}$.

Throughout this paper, let $(X, d)$ be a metric space with $n$ points, $1 \leq k \leq$ $n-2$ be an integer representing the number of faults allowed, and let $0<\epsilon<\frac{1}{2}$ be a constant. Without loss of generality, we also assume that the minimum interpoint distance of $X$ is strictly greater than 1 . We denote $\Delta:=\max _{x, y \in X} d(x, y)$ as the diameter of $X$.

Suppose $r>0$. The ball of radius $r$ centered at $x$ is $B(x, r):=\{y \in X$ : $d(x, y) \leq r\}$. We say that a cluster $C \subseteq X$ has radius at most $r$, if there exists $x \in C$ such that $C \subseteq B(x, r)$. Let $r_{2}>r_{1}>0$. The ring of inner radius $r_{1}$ and outer radius $r_{2}$ centered at $x$ is $R\left(x, r_{1}, r_{2}\right):=B\left(x, r_{2}\right) \backslash B\left(x, r_{1}\right)$.

A set $Y \subseteq X$ is an $r$-cover for $X$ if for any point $x \in X$ there is a point $y \in Y$ such that $d(x, y) \leq r$. A set $Y$ is an $r$-packing if for any pair of distinct points $y, y^{\prime} \in Y$, it holds that $d\left(y, y^{\prime}\right)>r$. We say that a set $Y \subseteq X$ is an $r$-net for $X$ if $Y$ is both an $r$-cover for $X$ and an $r$-packing. Note that if $X$ is finite, an $r$-net can be constructed greedily.

By recursively applying the definition of doubling dimension, we can get the following key proposition [11].

Proposition 1 (Nets Have Small Size). Let $R \geq 2 r>0$ and let $Y \subseteq X$ be an $r$-packing contained in a ball of radius $R$. Then, $|Y| \leq\left(\frac{R}{r}\right)^{2 \mathrm{dim}}$.

\section{Basic Construction of Sparse Fault-Tolerant Spanners}

In this section, we extend the $O(n)$-edge spanner construction in [4] and build a $k$-VFTS with $O(k n)$ edges. We construct $k+1$ sequences of hierarchical nets and assign each sequence with a distinct "color". Then based on the hierarchy of each color, we extract a net-tree. We show some properties similar to those in [3], and in addition we show that the fault-tolerance property can be established. Fault-Tolerant Hierarchical Nets. We color each point in $X$ with one of $k+1$ colors and let $X_{c}$ be the set of points with color $c$. For each color $c \in[k+1]$, we build a sequence of hierarchical nets of $\ell:=\left\lceil\log _{2} \Delta\right\rceil$ levels, $X_{c}=N_{0}^{c} \supseteq N_{1}^{c} \supseteq$ $\cdots \supseteq N_{\ell}^{c}$. We denote by $N_{i}:=\cup_{c \in[k+1]} N_{i}^{c}$ the set of all level- $i$ net points. Let $r_{i}:=2^{i}$ be the distance scale of level $i$. Fault-tolerant hierarchical nets should satisfy the following properties: 
1. Packing. For each $0 \leq i \leq \ell$ and $c \in[k+1], N_{i}^{c}$ is an $r_{i}$-packing;

2. Covering. For any $1 \leq i \leq \ell$, if $x \in X$ is not a net point in $N_{i}$, then for each color $c \in[k+1]$, there exists a net point $y_{c} \in N_{i}^{c}$ such that $d\left(y_{c}, x\right) \leq r_{i}$.

Construction. The hierarchical nets can be constructed in a top-down approach. Initially, each $N_{\ell}^{c}$ consists of a distinct point in $X$. Note that $k \leq n-2$ and hence the initialization is well defined. Also, the single point in $N_{\ell}^{c}$ is colored with $c$ and points not included in any cluster $N_{\ell}^{c}$ stay uncolored.

Suppose all nets on level $i+1$ have been built and we construct the level- $i$ nets as follows. For $c$ from 1 to $k+1$, let $U_{c}$ be the set of uncolored points when we start to build $N_{i}^{c}$, i.e., after finishing the construction of $N_{i}^{1}, N_{i}^{2}, \ldots, N_{i}^{c-1}$. We initialize $N_{i}^{c}:=N_{i+1}^{c}$, and extend $N_{i+1}^{c}$ to get $N_{i}^{c}$ by greedily adding points in $U_{c}$ to $N_{i}^{c}$ such that the resulting $N_{i}^{c}$ is an $r_{i}$-net for $U_{c}$; we color the points in $N_{i}^{c} \cap U_{c}$ with color $c$.

Note that the packing property and the covering property follow directly from the net construction.

Fault-Tolerant Net-Trees. For each color $c \in[k+1]$, we define a net-tree $T_{c}$, which spans all nodes in $X$ except nodes in the highest level $N_{\ell}$ with colors different from $c$. The construction is given in Algorithm 1. It follows from the construction that all internal nodes of $T_{c}$ have color $c$, and all points excluding $N_{\ell} \cup X_{c}$ are leaves of $T_{c}$.

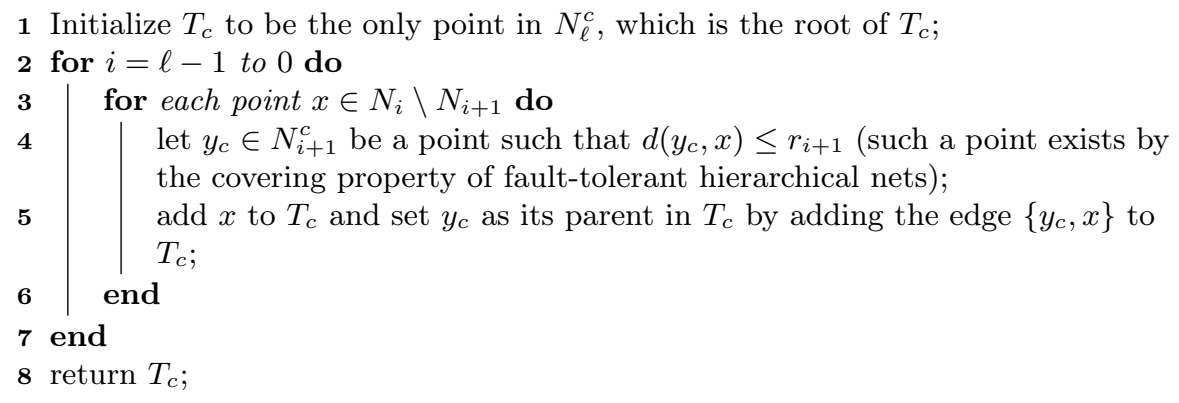

Algorithm 1: Construction of net-tree $T_{c}$ for color $c$

For any $c \in[k+1]$, a path $P=\left\{x_{0}, x_{1}, \ldots, x_{i}\right\}$ is called a $c$-path, if all edges on $P$ are contained in $T_{c}$. Note that for a $c$-path $P=\left\{x_{0}, x_{1}, \ldots, x_{i}\right\}$, any point $x_{j}$ with $0<j<i$ has degree at least 2 in $T_{c}$ and hence is an internal node. Thus, its color must be $c$. The length of $P$ is defined as length $(P):=\sum_{j=1}^{i} d\left(x_{i-1}, x_{i}\right)$. The following lemma shows that for any point $x \notin N_{\ell}$, any color $c \in[k+1]$ and any $0 \leq i \leq \ell$, there is a $c$-path from $x$ to some node in $N_{i}$ with length at most $2 r_{i}$.

Lemma 1 (Climbing Path in Net-tree). Let $T_{c}$ be a net-tree obtained above and let $x \in X \backslash N_{\ell}$ be a non-root point. For any $0 \leq i \leq \ell$, there exists a c-path $P_{i}$ 
starting from $x$ and ending at some net point $x_{i} \in N_{i}$, such that length $\left(P_{i}\right) \leq 2 \cdot r_{i}$. In addition, $x_{i}$ also has color $c$ if $x_{i} \neq x$.

Proof. Let $i^{*}(x)$ be the largest $i$ such that $x \in N_{i}$. For $0 \leq i \leq i^{*}(x)$, let $P_{i}=\{x\}$ and $x_{i}=x$. The conclusion holds trivially for $P_{i}$.

Now suppose $i^{*}(x)<i \leq \ell$ and we use induction on $i$. The base case is for $i=i^{*}(x)+1$. We let $x_{i}$ be $x$ 's parent in $T_{c}$ and let $P_{i}:=\left\{x, x_{i}\right\}$. Note that $P_{i}$ is a $c$-path. By the construction of $T_{c}$, we know that $x_{i} \in N_{i}^{c}$ and $d\left(x, x_{i}\right) \leq r_{i}$. Hence, $x_{i}$ has color $c$ and length $\left(P_{i}\right)=d\left(x, x_{i}\right) \leq r_{i} \leq 2 \cdot r_{i}$.

Suppose $i>i^{*}(x)+1$ and there exists an $x_{i-1}$ with color $c$, and a $c$-path $P_{i-1}=\left\{x, \ldots, x_{i-1}\right\}$ such that length $\left(P_{i-1}\right) \leq 2 \cdot r_{i-1}$. If $x_{i-1} \in N_{i}$, let $x_{i}=x_{i-1}$ and $P_{i}=P_{i-1}$. In this case, we have length $\left(P_{i}\right)=\operatorname{length}\left(P_{i-1}\right) \leq 2 \cdot r_{i-1} \leq 2 \cdot r_{i}$. Other properties of $P_{i}$ follow directly from the properties of $P_{i-1}$, and hence $P_{i}$ is a $c$-path.

Otherwise, $x_{i-1} \notin N_{i}$ and we know that $x_{i-1}$ is not the root of $T_{c}$ and let $x_{i}$ be $x_{i-1}$ 's parent in $T_{c}$. By our construction of net-trees, $x_{i} \in N_{i}^{c}$ and hence has color $c$; in addition, $d\left(x_{i-1}, x_{i}\right) \leq r_{i}$. We let $P_{i}=P_{i-1} \oplus x_{i}$, which is formed by appending $x_{i}$ to the end of $P_{i-1}$. Note that the edge $\left\{x_{i-1}, x_{i}\right\} \in T_{c}$ and hence $P_{i} \subseteq T_{c}$ is a $c$-path. Also, length $\left(P_{i}\right)=$ length $\left(P_{i-1}\right)+d\left(x_{i-1}, x_{i}\right) \leq 2 \cdot r_{i-1}+r_{i}=$ $2 \cdot r_{i}$.

Fault-Tolerant Spanners. We have added inter-level edges in the net-trees. Now we add edges connecting net points at the same level to achieve small stretch. Define $\gamma:=4+\frac{32}{\epsilon}$. For any $0 \leq i \leq \ell$, we call $\{x, y\}$ a cross edge at level $i$, iff $x$ and $y$ are both in $N_{i}$, and $d(x, y) \leq \gamma \cdot r_{i}$. (An edge can be a cross edge at more than one level.) We construct a spanner $H$ by taking the union of all edges in the net-trees of all colors, and all cross edges at all levels, and claim that $H$ is a $k$-VFTS with stretch at most $1+\epsilon$.

Lemma 2 (Fault-Tolerant Stretch). Let $S \subseteq X$ be any set with $|S| \leq k$. For any $x, y \in X \backslash S, d_{H \backslash S}(x, y) \leq(1+\epsilon) \cdot d(x, y)$.

Proof. Fix $x \neq y \in X \backslash S$ and suppose $r_{i}<d(x, y) \leq r_{i+1}$. Let $q$ be some integer such that $\frac{8}{2^{q}} \leq \epsilon<\frac{16}{2^{q}}$, say $q:=\left\lceil\log \frac{8}{\epsilon}\right\rceil$.

If $i \leq q-1$, then $d(x, y)<r_{i+1} \leq 2^{q}<\frac{16}{\epsilon}<\gamma \cdot r_{0}$. Hence, $\{x, y\}$ is a cross edge at level 0 and $d_{H \backslash S}(x, y)=d(x, y)$.

Now suppose $i \geq q$ and let $j:=i-q \geq 0$. Since $|S| \leq k$, there exists some $c \in[k+1]$ such that $X_{c} \cap S=\emptyset$. We first show that there exist $x^{\prime}$ and $y^{\prime}$ such that $d_{H \backslash S}\left(x, x^{\prime}\right) \leq 2 \cdot r_{j}$ and $d_{H \backslash S}\left(y, y^{\prime}\right) \leq 2 \cdot r_{j}$. Note that either the node $x$ is in $N_{j}$, or $i^{*}(x)<j$ and hence $x \notin N_{\ell}$. In the latter case, by Lemma 1 , there exists an $x_{j} \in N_{j}$ with color $c$ and a $c$-path $P$ connecting $x$ and $x_{j}$. We let $x^{\prime}=x$ and $x^{\prime}=x_{j}$ in respective cases. If $x^{\prime}=x$, then $d_{H \backslash S}\left(x, x^{\prime}\right)=d\left(x, x^{\prime}\right)=0 \leq 2 \cdot r_{j}$. Otherwise, since $P$ is a $c$-path and $x^{\prime}$ has color $c$, no point on $P$ is contained in $S$ and hence $P \subseteq H \backslash S$. Therefore, we have $d_{H \backslash S}\left(x, x^{\prime}\right) \leq 2 \cdot r_{j}$. Similarly, we can choose some $y^{\prime} \in N_{j} \backslash S$ such that $d_{H \backslash S}\left(y, y^{\prime}\right) \leq 2 \cdot r_{j}$.

Note that $d\left(x, x^{\prime}\right) \leq d_{H \backslash S}\left(x, x^{\prime}\right) \leq 2 r_{j}$ and $d\left(y, y^{\prime}\right) \leq d_{H \backslash S}\left(y, y^{\prime}\right) \leq 2 r_{j}$. Hence, we have $d\left(x^{\prime}, y^{\prime}\right) \leq d\left(x^{\prime}, x\right)+d(x, y)+d\left(y, y^{\prime}\right) \leq 2 r_{j}+r_{i+1}+2 r_{j}<$ 
$\left(4+\frac{32}{\epsilon}\right) \cdot r_{j}=\gamma \cdot r_{j}$. It follows that $\left\{x^{\prime}, y^{\prime}\right\}$ is a cross edge at level $j$ and thus $d_{H \backslash S}\left(x^{\prime}, y^{\prime}\right)=d\left(x^{\prime}, y^{\prime}\right)$.

Note that $d\left(x^{\prime}, y^{\prime}\right) \leq d\left(x^{\prime}, x\right)+d(x, y)+d\left(y, y^{\prime}\right) \leq 4 r_{j}+d(x, y)$, and $d_{H \backslash S}(x, y) \leq$ $d_{H \backslash S}\left(x, x^{\prime}\right)+d_{H \backslash S}\left(x^{\prime}, y^{\prime}\right)+d_{H \backslash S}\left(y^{\prime}, y\right)$. Hence we conclude that $d_{H \backslash S}(x, y) \leq$ $8 \cdot r_{j}+d(x, y)=\frac{8}{2^{q}} \cdot r_{i}+d(x, y) \leq(1+\epsilon) \cdot d(x, y)$.

Remark. Note that in the proof, there exists a color $c$ such that no node in $S$ has color $c$. Also, the spanner path in $H \backslash S$ is the concatenation of a path $P_{1}=\left\{x, \ldots, x^{\prime}\right\}$, a cross edge $\left\{x^{\prime}, y^{\prime}\right\}$, and a path $P_{2}=\left\{y^{\prime}, \ldots, y\right\}$, such that each of $P_{1}$ and $P_{2}$ is either a $c$-path or a trivial path with only one point. This property is useful in our later construction of $k$-VFTS with small hop-diameter. Bounding the Number of Edges. We show that the number of edges in $H$ is $O(k n)$. We actually show a stronger result: we can direct the edges in $H$ in a way such that every point has out-degree $O(k)$.

Lemma 3 (Bounding the Number of Edges). Let $H$ be the $(k, 1+\epsilon)$-VFTS we construct above. Then, the number of edges in $H$ is $\epsilon^{-O(\operatorname{dim})} \cdot k n$. Moreover, the edges of $H$ can be directed such that the out-degree of each point is bounded by $\epsilon^{-O(\operatorname{dim})} \cdot k$.

Proof. Note that the edges of $H$ come from two sources: the net-trees and cross edges; we bound them separately.

In any net-tree $T_{c}$, we direct the edge $\{x, p(x)\}$ from $x$ to $p(x)$, where $p(x)$ is the parent of $x$ in $T_{c}$. Note that every point has out-degree at most 1 in each tree, and hence the out-degree due to the net-tree edges is bounded by $k+1$.

Now we bound the out-degree due to the cross edges. Recall that $i^{*}(x)$ is the maximum $i$ such that $x \in N_{i}$. Given an edge $\{x, y\}$, we direct it from $x$ to $y$ if $i^{*}(x)<i^{*}(y)$, and direct it arbitrarily if $i^{*}(x)=i^{*}(y)$.

Fix $x \in X$. We bound the number of edges coming out of $x$ and going into some point with a fixed color $c$. Let $i:=i^{*}(x)$. For any directed edge $(x, y)$ such that $y$ has color $c$, we know that $i^{*}(y) \geq i^{*}(x)=i$ and hence $y \in N_{i}^{c}$. Note that the existence of a cross edge $\{x, y\}$ implies that $d(x, y) \leq \gamma \cdot r_{i}$. Also note that $N_{i}^{c}$ is an $r_{i}$-packing. Then, by Proposition 1 , the number of such edges is $\gamma^{O(\operatorname{dim})}=\left(4+\frac{32}{\epsilon}\right)^{O(\operatorname{dim})}$. Since there are at most $k+1$ colors, the number of cross edges coming out of $x$ is bounded by $(k+1) \cdot\left(4+\frac{32}{\epsilon}\right)^{O(\operatorname{dim})}=\epsilon^{-O(\operatorname{dim})} \cdot k$.

The upper bound on the number of edges follows directly from the analysis of out-degree.

\section{Achieving Small Hop-diameter}

In this section, we show that a technique similar to that in [4] can be used to reduce the hop-diameter of our basic $k$-VFTS.

Let $T$ be a tree metric with $n$ nodes. It is shown in [5,4] that for $m \geq 2 n$, we can add $m$ edges to $T$ to obtain a spanner $R$, such that for the unique tree path $P$ between $x$ and $y$ in $T$, there is a path $P^{\prime}$ in $R$ that connects $x$ and $y$ via at most $O(\alpha(m, n))$ nodes on $P$ (in the same order), where $\alpha(\cdot, \cdot)$ is defined below. By the triangle inequality, length $\left(P^{\prime}\right) \leq$ length $(P)$. 
Definition 2 (Ackermann's Function [17]). Let $A(i, j)$ be a function defined for integers $i, j \geq 0$ as the following.

$$
\begin{array}{lrl}
A(0, j) & =2 j & \text { for } j \geq 0 \\
A(i, 0) & =0, A(i, 1)=2 & \text { for } i \geq 1 \\
A(i, j) & =A(i-1, A(i, j-1)) & \text { for } i \geq 1, j \geq 2
\end{array}
$$

Define the function $\alpha(m, n):=\min \left\{i \mid i \geq 1, A\left(i, 4\left\lceil\frac{m}{n}\right\rceil\right)>\log _{2} n\right\}$.

Adding Edges to Reduce Hop-diameter. Let $H$ be the $(k, 1+\epsilon)$-VFTS constructed in Section 2. Now we show how to add edges to $H$ to reduce the hop-diameter. For each net-tree $T_{c}$, we use the technique in [5] to add $m$ edges to $T_{c}$ to get a spanner $R_{c}$ such that between any two points in $T_{c}$, there is a path between them in $R_{c}$ with $O(\alpha(m, n))$ hops which preserves their original path distance in $T_{c}$. Let $H^{\prime}$ denote the spanner constructed by taking the union of all edges in $R_{c}$ 's for all colors, and all cross edges at all levels. Hence, $H^{\prime}$ has $k\left(m+\epsilon^{-O(\operatorname{dim})} \cdot n\right)$ edges. We prove in the following lemma that $H^{\prime}$ has small hop-diameter.

Lemma 4 (Bounded Hop-Diameter). For $m \geq 2 n$, the spanner $H^{\prime}$ constructed above has $k\left(m+\epsilon^{-O(\mathrm{dim})} \cdot n\right)$ edges. Let $S \subseteq X$ be a set with $|S| \leq k$. For any pair $x, y \in X \backslash S$, there exists a path between $x$ and $y$ in $H^{\prime} \backslash S$ with $O(\alpha(m, n))$ hops, and the path has length at most $(1+\epsilon) \cdot d(x, y)$.

Proof. In Lemma 2, we have proved that there is a color $c$ such that no point in $S$ has color $c$, and the $(1+\epsilon)$-spanner path in $H \backslash S$ connecting $x$ and $y$ is a concatenation of a path $P_{1}$, a cross edge and a path $P_{2}$, where each of $P_{1}$ and $P_{2}$ is either a $c$-path or a trivial path consisting of only one point. Note that if $P_{1}$ is not a trivial path, it can be substituted by a path $P_{1}^{\prime}$ in $R_{c} \backslash S \subseteq H^{\prime} \backslash S$ consisting of $O(\alpha(m, n))$ hops. Similarly, $P_{2}$ can also be substitued by a path $P_{2}^{\prime}$ with $O(\alpha(m, n))$ hops. The new spanner path connecting $x$ and $y$ in $H^{\prime} \backslash S$ after the substitution has length at most $(1+\epsilon) \cdot d(x, y)$, as length $\left(P_{1}^{\prime}\right) \leq$ length $\left(P_{1}\right)$ and length $\left(P_{2}^{\prime}\right) \leq$ length $\left(P_{2}\right)$.

\section{Achieving Bounded Degree}

\subsection{Fault-tolerant Single-sink Spanners}

Our technique of reducing degrees in fault-tolerant spanners is based on singlesink spanners. Given a point $v \in X$, a spanner $H$ for $X$ is a $k$-vertex-faulttolerant $v$-single-sink $t$-spanner $((k, t, v)$-VFTssS $)$, if for any subset $S \subseteq X \backslash\{v\}$ with $|S| \leq k$, and any point $x \in X \backslash S$, it holds that $d_{H \backslash S}(v, x) \leq t \cdot d(v, x)$. Here, $t$ is called the root-stretch of $H$. In this section, we show a construction of a $(k, 1+\epsilon, v)$-VFTssS with maximum degree $O(k)$. Throughout this section, we assume a point $v \in X$ is given. Without loss of generality, we assume $0<\epsilon<\frac{1}{9}$ is a constant and build a $(k, 1+9 \epsilon, v)$-VFTssS. Our construction is based on a technique called ring-partition. 
Ring-Partition. Let $\ell=\left[\log _{\frac{1}{\epsilon}} \Delta\right]$ and $r_{i}=\frac{1}{\epsilon^{i}}$ with $i \in[\ell]$. For convenience, let $r_{0}=1$ (recall that we assume inter-point distances are larger than 1 ). Consider the rings, denoted by $R_{1}, \ldots, R_{\ell}$, where $R_{i}:=R\left(v, r_{i-1}, r_{i}\right)$. For convenience, let $R_{0}:=\{v\}$. The rings are pairwise disjoint and their union covers $X$. For each $i \in[\ell]$, we build an $\epsilon r_{i-1}$-net $N_{i}$ for $R_{i}$. By Proposition $1, N_{i}$ contains at most $\left(\frac{r_{i}}{\epsilon r_{i-1}}\right)^{2 \mathrm{dim}}=\epsilon^{-4 \mathrm{dim}}$ points. We denote this upper bound by $\Gamma:=\left\lceil\epsilon^{-4 \mathrm{dim}}\right\rceil$ and then we have $\left|N_{i}\right| \leq \Gamma$. Let $N:=\cup_{i>0} N_{i}$ be the set of net points. Then, for each net point $y \in N_{i}$, we construct a net cluster $C_{y}$, such that a point $x \in X$ is in $C_{y}$ iff $x$ is in $R_{i}$, and among all points in $N_{i}, y$ is the closest one to $x$ (breaking ties arbitrarily). For each $y \in N$, we arbitrarily choose $k+1$ portals $Q_{y} \subseteq C_{y}$ (if $\left|C_{y}\right|<k+1$, we let $\left.Q_{y}=C_{y}\right)$. Let $Q_{i}:=\cup_{y \in N_{i}} Q_{y}$ be the portals in $R_{i}$, and and $Q:=\cup_{y \in N} Q_{y}$ be the set of all portals. Note that $\left|Q_{i}\right| \leq(k+1) \cdot\left|N_{i}\right| \leq \Gamma \cdot(k+1)$.

We construct a $(k, 1+9 \epsilon, v)$-VFTssS in two stages. First, we add edges to connect portals to the root and obtain a $(k, 1+\epsilon, v)$-VFTssS for $Q \cup\{v\}$. Then, we add edges to connect the points in each cluster with their portals by short paths.

Connecting Portals to the Root. Assign each point $q \in Q$ a unique identifier $\operatorname{id}(q) \in[|Q|]$, such that for any $q \in R_{i}$ and $q^{\prime} \in R_{j}$ with $i<j$, it holds that $\operatorname{id}(q)<\operatorname{id}\left(q^{\prime}\right)$; also let $\operatorname{id}(v)=0$. In other words, points closer to the root $v$ have smaller identifiers. We divide the points in $Q$ into groups of size $k+1$. Specifically, let $A_{j}:=\{q \in Q \mid(j-1) \cdot(k+1)+1 \leq \mathrm{id}(q) \leq j \cdot(k+1)\}$ for $j \geq 1$. The edges to connect portals with the root are added as follows.

- For $q \in Q$ with $1 \leq \mathrm{id}(q) \leq(2 \Gamma+1) \cdot(k+1)$, add an edge $\{q, v\}$. Let $E_{0}$ denote the set of such edges.

- For $j>2 \Gamma+1$, we add an edge between every point in $A_{j}$ and every point in $A_{j-2 \Gamma-1}$, and let $E_{j}$ denote the set of such edges, i.e. $E_{j}:=\{\{x, y\} \mid x \in$ $A_{j}$ and $\left.y \in A_{j-2 \Gamma-1}\right\}$.

Define $\tilde{E}:=E_{0} \cup\left(\cup_{j>2 \Gamma+1} E_{j}\right)$ and $\tilde{H}:=(Q \cup\{v\}, \tilde{E})$. Note that the degree of $v$ in $\tilde{H}$ is at most $(2 \Gamma+1) \cdot(k+1)=\epsilon^{-O(\operatorname{dim})} \cdot k$, and the degree of any point $q \in Q$ is at most $2(k+1)=O(k)$.

Lemma 5 (Edges Connect Portals at least Two Levels Apart). Let $q \in$ $Q$, and let $\{q, x\} \in \tilde{E}$ be an edge with $\operatorname{id}(x)<\operatorname{id}(q)$. Then, either $x=v$ or there exist $i>2$ and $0<i^{\prime} \leq i-2$ such that $q \in Q_{i}$ and $x \in Q_{i^{\prime}}$.

Proof. We first consider $\{q, x\}$ with $q \in Q_{1} \cup Q_{2}$. Since $\left|Q_{1}\right| \leq \Gamma \cdot(k+1)$ and $\left|Q_{2}\right| \leq \Gamma \cdot(k+1)$, it holds that id $(q) \leq 2 \Gamma \cdot(k+1) \leq(2 \Gamma+1) \cdot(k+1)$. Hence, $q$ is connected to the root and $x=v$.

Now consider the case that $q \in Q_{i}$ for some $i>2$. If $x=v$, then we are done. Now suppose $x \neq v$. Then, we know that $q \in A_{j}$ for some $j>2 \Gamma+1$ and $x \in A_{j-2 \Gamma-1}$. Note that there are exactly $2 \Gamma \cdot(k+1)$ points in $A_{j-2 \Gamma} \cup \cdots \cup A_{j-1}$, and hence id $(x)<\operatorname{id}(q)-2 \Gamma \cdot(k+1)$. On the other hand, since there are at most $2 \Gamma \cdot(k+1)$ points in $Q_{i} \cup Q_{i-1}, x$ cannot be in $Q_{i} \cup Q_{i-1}$. Hence, $x \in Q_{i^{\prime}}$ for some $i^{\prime} \leq i-2$.

Lemma 6. Let $S \subseteq X \backslash\{v\}$ be a set of at most $k$ points. Then, for any point $q \in Q \backslash S, d_{\tilde{H} \backslash S}(v, q) \leq(1+3 \epsilon) \cdot d(v, q)$. 
Proof. We use induction on id $(q)$. For $q \in Q \backslash S$ with $1 \leq \mathrm{id}(q) \leq(2 \Gamma+1) \cdot(k+1)$, we know that $q$ is connected to $v$ in $\tilde{H}$ and hence $d_{\tilde{H}}(v, q)=d(v, q)$.

Now suppose id $(q)>(2 \Gamma+1) \cdot(k+1)$ and for any point $q^{\prime} \in Q \backslash S$ with $\operatorname{id}\left(q^{\prime}\right)<\operatorname{id}(q)$, it holds that $d_{\tilde{H} \backslash S}\left(v, q^{\prime}\right) \leq(1+3 \epsilon) d\left(v, q^{\prime}\right)$. Let $j>2 \Gamma+1$ be such that $q \in A_{j}$. From the construction of $\tilde{H}$, we know that for any point $p \in$ $A_{j-2 \Gamma-1}$, there exists an edge $\{p, q\} \in \tilde{H}$. Since $A_{j-2 \Gamma-1}$ contains $k+1$ points, there exists a functioning point $p^{*} \notin S$ in $A_{j-2 \Gamma-1}$. Note that $\operatorname{id}\left(p^{*}\right)<\operatorname{id}(q)$. Hence, we have

$$
d_{\tilde{H} \backslash S}\left(v, p^{*}\right) \leq(1+3 \epsilon) d\left(v, p^{*}\right)
$$

by induction hypothesis.

Note that id $(q)>(2 \Gamma+1) \cdot(k+1)$ implies $q \in Q_{i}$ for some $i>2$. By Lemma 5 , we have $p^{*} \in Q_{i^{\prime}} \subseteq R_{i^{\prime}}$ for some $0<i^{\prime} \leq i-2$. Hence,

$$
d\left(v, p^{*}\right) \leq r_{i^{\prime}}=\epsilon r_{i^{\prime}+1} \leq \epsilon r_{i-1}<\epsilon d(v, q)
$$

By the triangle inequality, $d_{\tilde{H} \backslash S}(v, q) \leq d_{\tilde{H} \backslash S}\left(v, p^{*}\right)+d\left(p^{*}, q\right) \leq d_{\tilde{H} \backslash S}\left(v, p^{*}\right)+$ $d\left(p^{*}, v\right)+d(v, q)$, which by (1) and (2) is at most $(1+\epsilon(2+3 \epsilon)) d(v, q) \leq(1+$ $3 \epsilon) d(v, q)$, where the last inequality holds when $\epsilon \leq \frac{1}{3}$.

Connecting Points in Clusters to Portals. Fix $i \in[\ell]$ and a point $y \in N_{i}$. Recall that $C_{y}$ denotes the net cluster centered at $y$, whose radius is at most $r:=\epsilon \cdot r_{i-1}$, and $Q_{y}$ is the set of portals for $C_{y}$. We call the portals in $Q_{y}$ $r$-portals since they are portals for clusters with radius at most $r$. We define a procedure $\operatorname{Add}\left(C_{y}, Q_{y}, r\right)$ which adds edges to connect points in $C_{y}$ with portals in $Q_{y}$.

1. Sub-clustering. We return immediately if $C_{y}=Q_{y}$. Suppose $C_{y} \neq Q_{y}$. We build an $\frac{r}{2}$-net $\widehat{N}$ for $C_{y} \backslash Q_{y}$. Recall that $r$ is an upper bound on $C_{y}$ 's radius. By Proposition $1,|\widehat{N}| \leq 4^{\operatorname{dim}}$. Then for each node $z \in \widehat{N}$, we construct a cluster $\widehat{C}_{z}$, such that a point $x \in C_{y}$ is in $\widehat{C}_{z}$ iff $x$ is in $C_{y} \backslash Q_{y}$, and among all points of $\widehat{N}, z$ is the closest one to $x$ (breaking ties arbitrarily).

2. Connecting sub-portals. For each sub-cluster $\widehat{C}_{z}$, we arbitrarily select $k+1$ sub-portals $\widehat{Q}_{z}$ (called $\frac{r}{2}$-portals) in $\widehat{C}_{z}$ (select all points if $\left|\widehat{C}_{z}\right|<k+1$ ). Then, for each sub-portal in $\widehat{Q}_{z}$ and each portal in $Q_{y}$, we add an edge between them. Note that since $\widehat{N} \leq 4^{\mathrm{dim}}$, and thus each portal $q \in Q_{y}$ is connected with at most $4^{\mathrm{dim}} \cdot(k+1)=2^{O(\operatorname{dim})} \cdot k$ sub-portals.

3. Recursion. For every $z \in \widehat{N}$, recursively call $\operatorname{Add}\left(\widehat{C}_{z}, \widehat{Q}_{z}, \frac{r}{2}\right)$.

Let $\widehat{H}_{y}$ be the resulting spanner returned by $\operatorname{Add}\left(C_{y}, Q_{y}, r\right)$. We have the following lemma.

Lemma 7. Let $S \subseteq X$ be a set of at most $k$ points. For any $x \in C_{y} \backslash\left(Q_{y} \cup S\right)$ and any $r$-portal $q \in Q_{y} \backslash S$, it holds that $d_{\widehat{H}_{y} \backslash S}(x, q) \leq 2 r$. 
Proof. Suppose that $x$ is an $\frac{r}{2^{i}}$-portal. Note that $x$ is connected to $k+1$ distinct $\frac{r}{2^{i-1}}$-portals, and at least one of them must be functioning (i.e., not in $S$ ). We let $x_{i-1}$ be such a $\frac{r}{2^{i-1}}$-portal. Using this argument, we can find a sequence of portals $x=x_{i}, x_{i-1}, x_{i-2}, \ldots, x_{0}=q$, such that for all $0 \leq j \leq i, x_{j} \notin S$ is an $\frac{r}{2^{j}}$-portal. In addition, for $j \in[i],\left\{x_{j}, x_{j-1}\right\} \in \widehat{H}_{y}$ and $d\left(x_{j}, x_{j-1}\right) \leq \frac{r}{2^{j-1}}$. Hence, $d_{\widehat{H}_{y} \backslash S}(x, q) \leq \sum_{j=1}^{i} \frac{r}{2^{j-1}} \leq 2 r$.

Obtaining the $(k, 1+9 \epsilon, v)$-VFTssS. Our final $(k, 1+9 \epsilon, v)$-VFTssS, denoted by $H_{v}$, is the union of $\tilde{H}$ and $\widehat{H}_{y}$ 's for all $y \in N$. Note that the degree of $v$ is bounded by $\epsilon^{-O(\operatorname{dim})} \cdot k$ and the degree of any other point in $H_{v}$ is bounded by $2^{O(\operatorname{dim})} \cdot k$. It remains to show that $H_{v}$ has root-stretch at most $1+9 \epsilon$ under the failure of at most $k$ points.

Lemma 8. Let $S \subseteq X \backslash\{v\}$ be a set of at most $k$ points. For any $x \in X \backslash S$, $d_{H_{v}}(v, x) \leq(1+9 \epsilon) \cdot d(v, x)$.

Proof. Suppose $x \neq v$. Otherwise the conclusion holds trivially. Let $y \in N$ be the net point covering $x$, i.e., $x \in C_{y}$, and let $R_{i}$ be the ring that contains $C_{y}$.

If $x$ is a portal for $C_{y}$, then by Lemma 6 , we know that $d_{\tilde{H} \backslash S}(v, x) \leq(1+$ $3 \epsilon) d(v, x)$ and hence $d_{H_{v} \backslash S}(v, x) \leq d_{\tilde{H} \backslash S}(v, x) \leq(1+9 \epsilon) d(v, x)$.

Otherwise, $Q_{y} \neq C_{y}$ and hence $\left|Q_{y}\right|=k+1$. Therefore, there must be some $q \in Q_{y}$ which is functioning. Let $r:=\epsilon r_{i-1}$ be an upper bound on the radius of $C_{y}$. From the construction of the ring-partition, we know that $d(q, x) \leq r$ and $r \leq \epsilon \cdot d(v, x)$. By Lemma 7, it holds that $d_{\widehat{H}_{y} \backslash S}(q, x) \leq 2 r$. Hence, $d_{H_{v} \backslash S}(v, x) \leq$ $d_{H_{v} \backslash S}(v, q)+d_{H_{v} \backslash S}(q, x) \leq d_{\tilde{H} \backslash S}(v, q)+d_{\widehat{H}_{y} \backslash S}(q, x) \leq(1+3 \epsilon) d(v, q)+2 r \leq$ $(1+3 \epsilon)(d(v, x)+d(x, q))+2 r \leq(1+3 \epsilon) d(v, x)+6 r \leq(1+9 \epsilon) d(v, x)$.

\section{$4.2(k, 1+\epsilon)$-VFTS with Bounded Degree}

Now we construct a $(k, 1+\epsilon)$-VFTS with bounded degree as follows. We first construct a basic $\left(k, 1+\frac{\epsilon}{3}\right)$-VFTS for $X$ with $O(k n)$ edges, and denote it by $H_{0}$. Recall that the edges of $H_{0}$ can be directed such that the out-degree of each point in $H_{0}$ is $\epsilon^{-O(\operatorname{dim})} \cdot k$. Denote an edge $\{x, y\}$ by $(x, y)$ if it is directed from $x$ to $y$ in $H_{0}$. For any point $x \in X$, we let $N_{i n}(x):=\left\{y \in X \mid(y, x) \in H_{0}\right\}$, and build a $\left(k, 1+\frac{\epsilon}{3}, x\right)$-VFTssS $H_{x}$ for $N_{i n}(x) \cup\{x\}$. We take the spanner $H:=\cup_{x \in X} H_{x}$, and show that $H$ is a $(k, 1+\epsilon)$-VFTS with maximum degree $O\left(k^{2}\right)$.

Lemma 9. For $0<\epsilon \leq \frac{1}{2}, H$ is a $(k, 1+\epsilon)$-VFTS, in which the degree of any point $x \in X$ is $\epsilon^{-O(\operatorname{dim})} \cdot k^{2}$. Consequently, $H$ has $\epsilon^{-O(\operatorname{dim})} \cdot k^{2} n$ edges.

Proof. We first prove that $H$ is a $(k, 1+\epsilon)$-VFTS. Let $S \subseteq X$ be a set of at most $k$ points. Since $H_{0}$ is a $\left(k, 1+\frac{\epsilon}{3}\right)$-VFTS, for any $x, y \in X \backslash S$, there exists a $\left(1+\frac{\epsilon}{3}\right)$-spanner path $P_{0}$ in $H_{0} \backslash S$ between $x$ and $y$.

For each edge $\{u, v\} \in P_{0}$, suppose the edge is directed as $(u, v)$. Then, since $H_{v}$ is a $\left(k, 1+\frac{\epsilon}{3}, v\right)$-VFTssS and $u, v$ are both funcitoning, there is a $\left(1+\frac{\epsilon}{3}\right)$ spanner path $P_{u v} \subseteq H_{v} \backslash S$ between $u$ and $v$. Let $P$ denote the concatenation of 
$P_{u v}$ 's for all edges $\{u, v\} \in P_{0}$. Then, $P$ is contained in $H \backslash S$ and is a spanner path between $x$ and $y$ with stretch at most $\left(1+\frac{\epsilon}{3}\right)^{2} \leq 1+\epsilon$.

Next we bound the degree of an arbitrary point $x \in X$ in $H$. The edges incident to $x$ in $H$ are contained in $H_{x}$ and $H_{y}$ 's such that the edge $\{x, y\}$ is directed from $x$ to $y$ in $H_{0}$. Note that the number of $H_{y}$ 's involving $x$ is bounded by the out-degree of $x$ in $H_{0}$, which is $\epsilon^{-O(\operatorname{dim})} \cdot k$. Also recall that the degree of $x$ in $H_{x}$ is $\epsilon^{-O(\operatorname{dim})} \cdot k$ and the degree of $x$ in each $H_{y}$ is $2^{O(\operatorname{dim})} \cdot k$. Hence, we conclude that the degree of $x$ in $H$ is $\epsilon^{-O(\operatorname{dim})} \cdot k^{2}$.

The upper bound on the number of edges follows directly from the degree analysis.

\section{References}

1. S. Arya, G. Das, D. M. Mount, J. S. Salowe, and M. H. M. Smid. Euclidean spanners: short, thin, and lanky. In STOC, pages 489-498, 1995.

2. P. B. Callahan and S. R. Kosaraju. Faster algorithms for some geometric graph problems in higher dimensions. In SODA, pages 291-300, 1993.

3. H. T.-H. Chan, A. Gupta, B. M. Maggs, and S. Zhou. On hierarchical routing in doubling metrics. In $S O D A$, pages 762-771, 2005.

4. T.-H. H. Chan and A. Gupta. Small hop-diameter sparse spanners for doubling metrics. Discrete \& Computational Geometry, 41(1):28-44, 2009.

5. B. Chazelle. Computing on a free tree via complexity-preserving mappings. Algorithmica, 2:337-361, 1987.

6. S. Chechik, M. Langberg, D. Peleg, and L. Roditty. Fault-tolerant spanners for general graphs. In STOC, pages 435-444, 2009.

7. A. Czumaj and H. Zhao. Fault-tolerant geometric spanners. Discrete \& Computational Geometry, 32(2):207-230, 2004.

8. G. Das and G. Narasimhan. A fast algorithm for constructing sparse euclidean spanners. In Symposium on Computational Geometry, pages 132-139, 1994.

9. M. Dinitz and R. Krauthgamer. Fault-tolerant spanners: better and simpler. In PODC, pages 169-178, 2011.

10. L.-A. Gottlieb and L. Roditty. An optimal dynamic spanner for doubling metric spaces. In ESA, pages 478-489, 2008.

11. A. Gupta, R. Krauthgamer, and J. R. Lee. Bounded geometries, fractals, and low-distortion embeddings. In FOCS, pages 534-543, 2003.

12. S. Har-Peled and M. Mendel. Fast construction of nets in low dimensional metrics, and their applications. In Symposium on Computational Geometry, pages 150-158, 2005.

13. C. Levcopoulos, G. Narasimhan, and M. H. M. Smid. Efficient algorithms for constructing fault-tolerant geometric spanners. In STOC, pages 186-195, 1998.

14. T. Lukovszki. New results of fault tolerant geometric spanners. In WADS, pages 193-204, 1999.

15. G. Narasimhan and M. H. M. Smid. Geometric spanner networks. Cambridge University Press, 2007.

16. S. Solomon and M. Elkin. Balancing degree, diameter and weight in euclidean spanners. In ESA (1), pages 48-59, 2010.

17. R. E. Tarjan. Efficiency of a good but not linear set union algorithm. J. ACM, $22(2): 215-225,1975$. 